\title{
Review of Structures in the Energy Dependence of Hadronic Observables
}

\section{Blume*}

Institut für Kernphysik, J.W. Goethe Universität, Frankfurt am Main, Germany

E-mail: blumedikf.uni-frankfurt.de

\begin{abstract}
The energy dependence of various hadronic observables is reviewed. The study of their evolution from AGS over SPS to the highest RHIC energy reveals interesting features, which might locate a possible onset of deconfinement. These observables include transverse spectra of different particle types and their total multiplicities, as well as elliptic flow. In this context especially the observation of a maximum of the strangeness to pion ratio is of particular interest, since on one hand it has been predicted as a signal for the onset of deconfinement but on the other hand also statistical model calculations exhibit qualitatively similar structures. The sharpness of these features is however not reproduced by hadronic scenarios. The significance of these structures will be discussed in this contribution. Other observables, such as radius parameters from BoseEinstein correlations, on the other hand do not exhibit any structure in their energy dependence.
\end{abstract}

The 3rd edition of the International Workshop — The Critical Point and Onset of Deconfinement July 3-7 2006

Galileo Galilei Institute, Florence, Italy

\footnotetext{
* Speaker.
} 

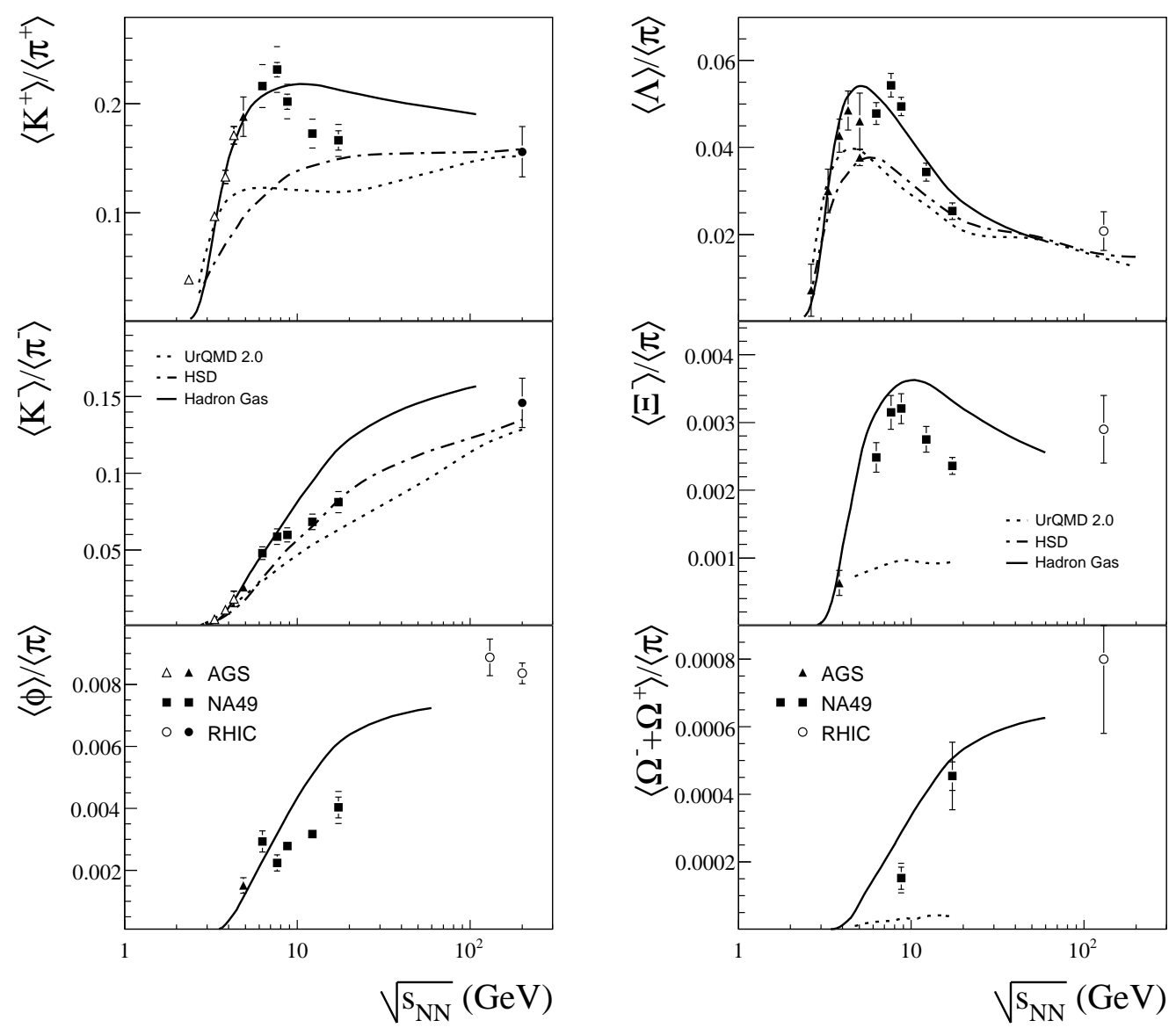

Figure 1: The energy dependence of the $4 \pi$-yields of strange hadrons, normalized to the pion yields, in central $\mathrm{Pb}+\mathrm{Pb} / \mathrm{Au}+\mathrm{Au}$ collisions [1]]. The data are compared to string hadronic models (UrQMD 2.0 [5]: dotted lines, HSD [6]: dashed-dotted lines) and a statistical hadron gas model [7], assuming full chemical equilibrium (solid line).

\section{Introduction}

Since the chemical freeze-out points approach the phase boundary line as predicted by lattice QCD already at top SPS energies, it is a reasonable conjecture that the transition to a quark-gluon plasma is likely to happen in the SPS energy regime (i.e. $\sqrt{s_{\mathrm{NN}}}=6-17.3 \mathrm{GeV}$ ). Also the energy density, which is achieved at top SPS beam energies, should be sufficiently high for a QGP formation. Therefore, a study of the energy dependence of heavy ion collisions might help to identify a possible phase transition and to localize the center-of-mass energy, where the phase boundary is reached first. In such a study one should look for any kind of "non-smooth" behaviour in the energy dependence of different observables. The most prominent examples of such kind of structures have been reported by the NA49 collaboration [2]: A pronounced maximum in the $\left\langle\mathrm{K}^{+}\right\rangle /\left\langle\pi^{+}\right\rangle$-ratio, and sudden changes in the energy dependence of the slope parameters of kaons and of pion production. In [3] two of these observations have been predicted as a signature for the onset of deconfinement. The questions that will be addressed in this contribution are: Are there any other structures in the 

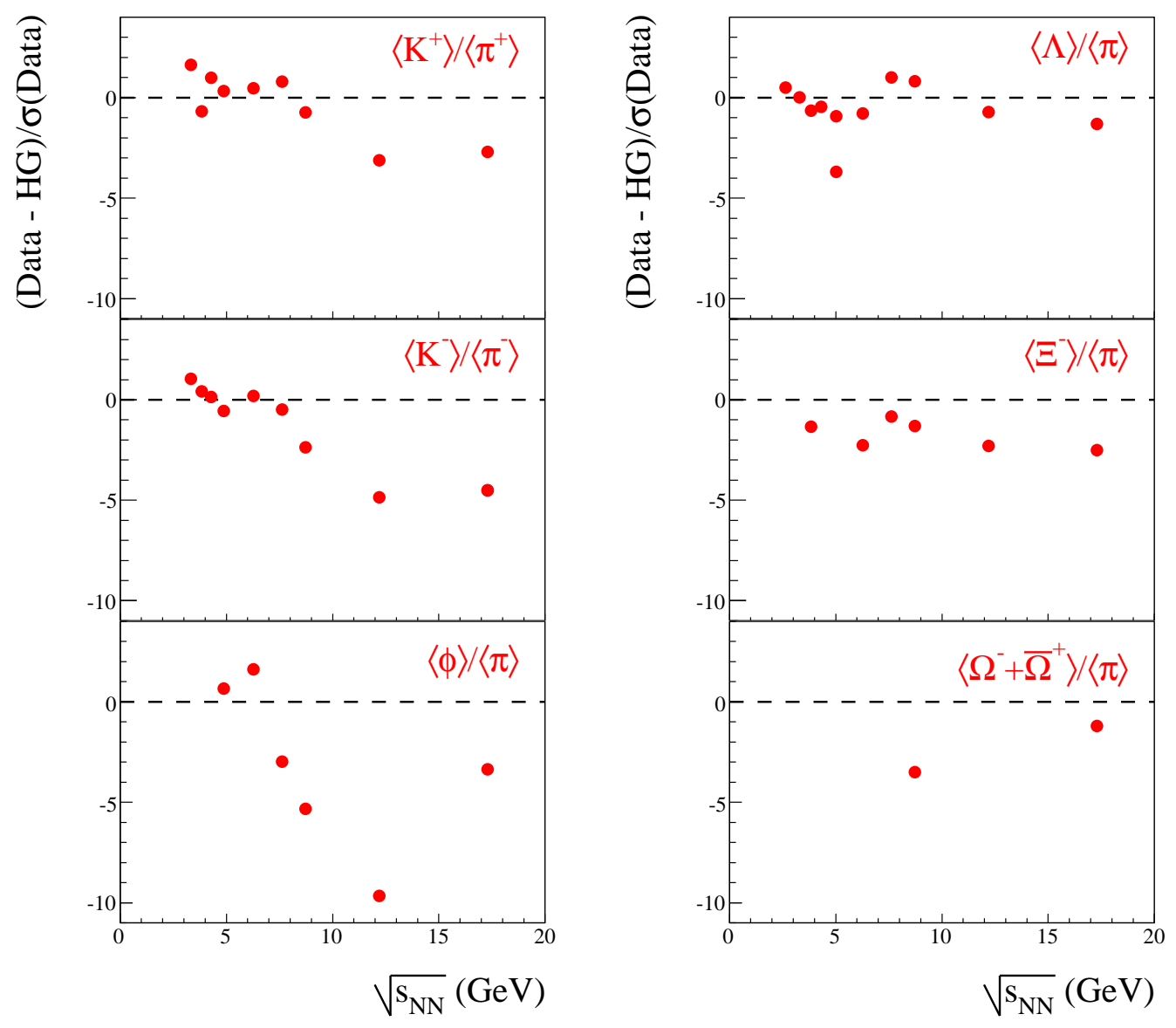

Figure 2: The difference of the measured particle ratios to the predictions of a fully equilibrated hadron gas [7] relative to the total error.

energy dependence of hadronic observables and what is the significance of any of these? Hadronic models (transport models like UrQMD [5] and HSD [6] or statistical models) provide an important baseline for a comparsion and help to identify "trivial" structures. One important aspect in this context is the fact that the fireball produced in heavy ion collisions changes its nature from being baryon dominated at lower energies to meson dominated at higher energies. In the framework of a statistical hadron gas model this transition was located around $\sqrt{s_{\mathrm{NN}}} \approx 8 \mathrm{GeV}$ which coincides with a maximum of the relative strangeness production [\#]. Therefore any kind of structure observed in the data has to be weighted against structures expected from this hadronic scenario.

\section{Particle Yields}

In Fig. 11 the energy dependence of the total multiplicities for a variety of strange hadrons, normalized to the pion yield, as measured by NA49 and various AGS and RHIC experiments [1], is summarized and compared to model predictions. Generally, it can be stated that the string hadronic models UrQMD [5] and HSD [6] do not provide a good description of the data points. Especially the $\Xi$ and $\Omega$ production is substantially underestimated and the maximum in the $\left\langle\mathrm{K}^{+}\right\rangle /\left\langle\pi^{+}\right\rangle$ratio is 


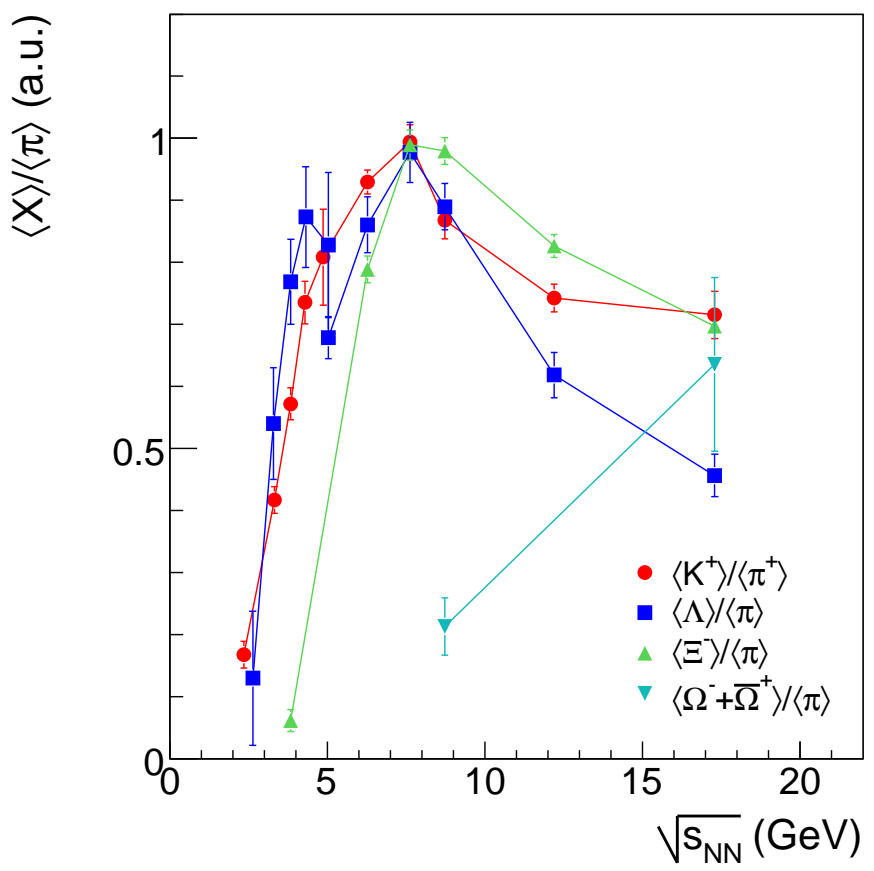

Figure 3: The energy dependence of the $4 \pi$-yields of strange hadrons, normalized to the pion yields, in central $\mathrm{Pb}+\mathrm{Pb} / \mathrm{Au}+\mathrm{Au}$ collisions. The ratios were scaled such that the maximum value is unity.

not reproduced. The basic trend of the $\left\langle\mathrm{K}^{-}\right\rangle /\left\langle\pi^{-}\right\rangle$and the $\langle\Lambda\rangle /\langle\pi\rangle$ ratios is on the other hand better described by the transport models, although discrepancies remain. A statistical hadron gas model assuming full chemical equilibrium [7], on the other hand, provides a better overall description of the measurements. This kind of model implictly takes into account the already mentioned transition from a baryon to a meson dominated system. However, also here clear differences to the NA49 measurements exist. These require the introduction of an energy dependent strangeness saturation factor $\gamma_{S}[8]$, in order to capture the structures in the energy dependence of most particle species (not shown here).

Since the full chemical equilbrium assumption in principle provides a relatively well defined theoretical baseline that is also qualitatively describing the trend of the measurements, it is worthwhile to look at the differences in more detail in order to see whether there are any common features. These are summarized in Fig. 2. As the left panel shows, both, charged kaons as well as $\phi$ mesons deviate by more than three times the total error from the statistical model prediction for $\sqrt{s_{\mathrm{NN}}}>7-8 \mathrm{GeV}$. The situation is less clear for $\Lambda$ and hyperons (Fig. 2 , right panel). While the $\langle\Lambda\rangle /\langle\pi\rangle$ ratios generally agree within the total error, there might be also an indication for a departure from the full equilibrium assumption for the $\left\langle\Xi^{-}\right\rangle /\langle\pi\rangle$ ratios, although not as obvious as for the kaons and $\phi$. The $\left\langle\Omega^{-}+\bar{\Omega}^{+}\right\rangle /\langle\pi\rangle$ ratio, on the other hand, seems to deviate stronger at the lower beam energy. However, for a more systematic evaluation of its energy dependence more data at different energies would be needed. Especially the question, whether the difference of the measured $\left\langle\Omega^{-}+\bar{\Omega}^{+}\right\rangle /\langle\pi\rangle$ ratio to the statistical model prediction increases towards even lower energies, or remains close to it (as it seems to be the case for the $\left\langle\Xi^{-}\right\rangle /\langle\pi\rangle$ ratio) would 

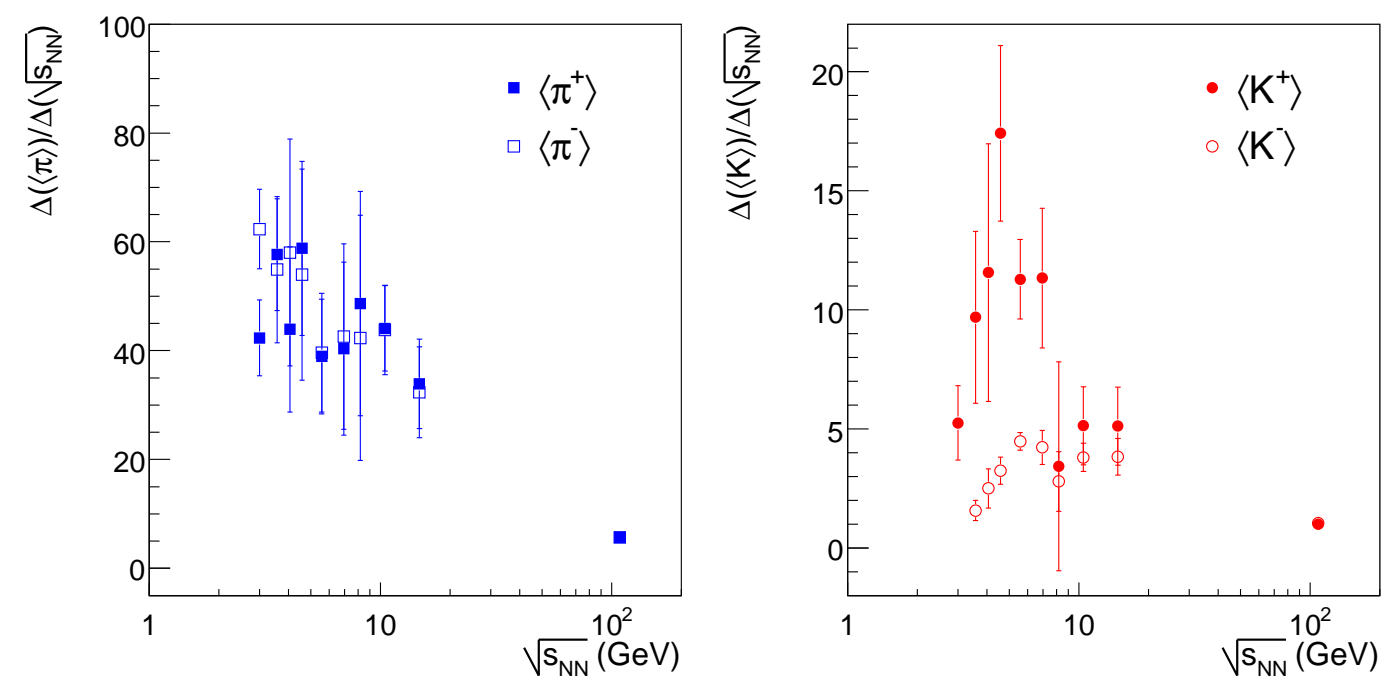

Figure 4: The local slopes of the energy dependencies of charged pions (left panel) $\Delta\langle\pi\rangle / \Delta \sqrt{s_{\mathrm{NN}}}$ and charged kaons (right panel) $\Delta\langle\mathrm{K}\rangle / \Delta \sqrt{s_{\mathrm{NN}}}$ as a function of $\sqrt{s_{\mathrm{NN}}}$.

be of particular interest. The first scenario would be expected if a dynamical equilibration of a heavy particle like the $\Omega$ is only possible at higher energies where the system is close to the phase boundary [9]. The second would rather indicate that particle production via the strong interaction always follows the maximum entropy principle.

As has been pointed out in [П], one of the predictions of the statistical model is that the position of the maxima in the energy dependence of the strange particles to pion ratios should depend on the strangeness content and the baryon number. In [ $₫$ the following positions are predicted: $\sqrt{s_{\mathrm{NN}}}=10.8 \mathrm{GeV}\left(\mathrm{K}^{+} / \pi^{+}\right), 5.1 \mathrm{GeV}(\Lambda / \pi), 10.2 \mathrm{GeV}\left(\Xi^{-} / \pi^{-}\right), 27.0 \mathrm{GeV}\left(\Omega^{-} / \pi^{-}\right)$. Figure 3 summarizes the current situation on the $4 \pi$ ratios, as also shown in Fig. 1. They were normalized to unity at the maximum in order to allow for a direct comparison of their positions. While the position of the maximum for the $\left\langle\mathrm{K}^{+}\right\rangle /\left\langle\pi^{+}\right\rangle$and $\langle\Lambda\rangle /\langle\pi\rangle$ ratios seems to coincide, it appears to be shifted a bit to higher values for the $\left\langle\Xi^{-}\right\rangle /\langle\pi\rangle$ ratio. However, both, the maximum for $\left\langle\mathrm{K}^{+}\right\rangle /\left\langle\pi^{+}\right\rangle$ and for $\left\langle\Xi^{-}\right\rangle /\langle\pi\rangle$ are at smaller values of $\sqrt{s_{\mathrm{NN}}}$ than predicted by the statistical model. For the $\left\langle\Omega^{-}+\bar{\Omega}^{+}\right\rangle /\langle\pi\rangle$ ratio no maximum can be observed, but rather a continous rise (see also right panel of Fig. 四).

One possible way to investigate whether there actually is any non-smooth behaviour in the energy dependencies of particle yields, is the study of their local slope:

$$
\frac{\Delta(N)}{\Delta\left(\sqrt{s_{\mathrm{NN}}}\right)}=\frac{N_{\mathrm{i}+1}-N_{\mathrm{i}}}{\sqrt{s_{\mathrm{NN} \mathrm{i}+1}}-\sqrt{s_{\mathrm{NN} \mathrm{i}}}}
$$

This quantity is shown in Fig. $\emptyset$ for charged pions and kaons, for which the errors of the measurements are small enough to allow its calculation with reasonable errors. The values of the local slopes have been derived from the same data sets as shown in Fig. 1. For the pions it decreases with increasing center-of-mass energy without exhibiting any significant structure. For kaons, on the other hand, there are indications for sudden changes in the energy dependence around 

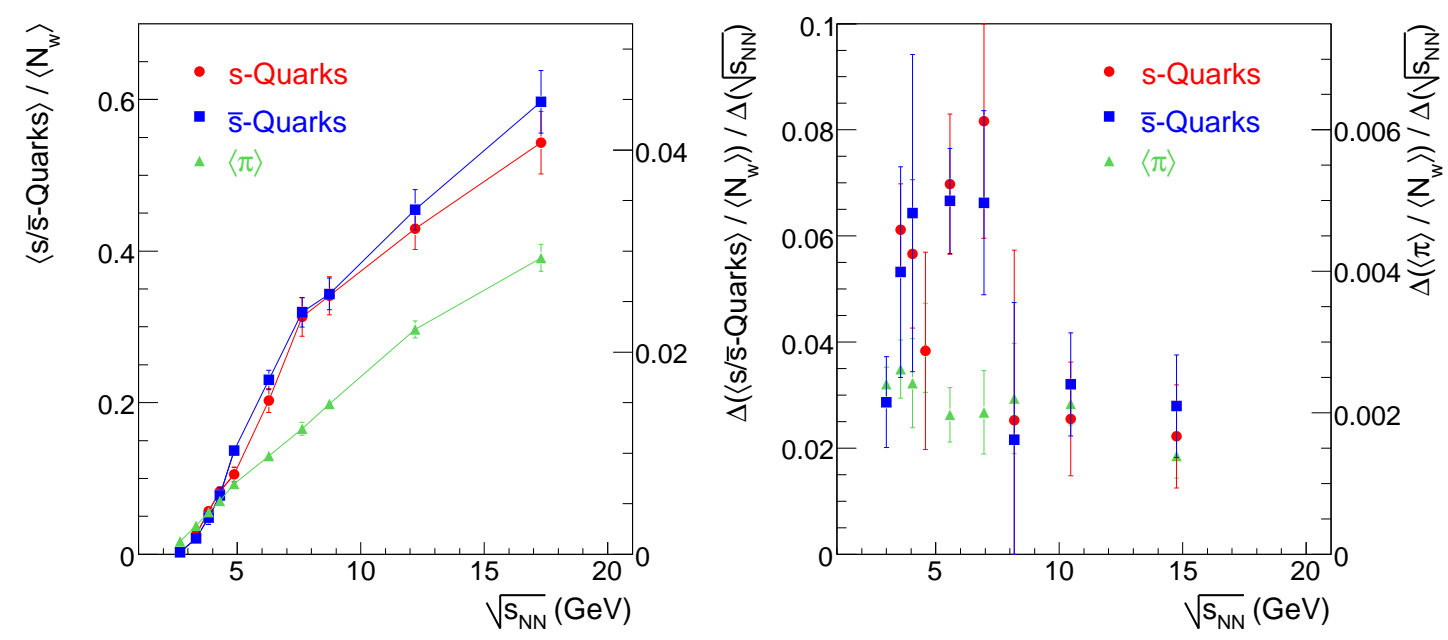

Figure 5: Left: The total number of strange quarks and anti-quarks as carried by kaons and hyperons together with the total number of pions, both normalized to the number of wounded nucleons $\left\langle N_{\mathrm{w}}\right\rangle$, versus the collision energy for central $\mathrm{Pb}+\mathrm{Pb}(\mathrm{Au}+\mathrm{Au})$ reactions [11]. Right: The local slope of the energy dependencies of strange (anti-)quarks $\Delta\left\langle s / \bar{s}\right.$-Quarks $\left./\left\langle N_{\mathrm{W}}\right\rangle\right\rangle / \Delta \sqrt{s_{\mathrm{NN}}}$ and pions $\Delta\left\langle\pi /\left\langle N_{\mathrm{w}}\right\rangle\right\rangle / \Delta \sqrt{s_{\mathrm{NN}}}$.

$\sqrt{s_{\mathrm{NN}}}=5-7 \mathrm{GeV}$. The local slope is clearly higher here compared to higher energies for the $\mathrm{K}^{+}$. For the $\mathrm{K}^{-}$it seems to increase until $\sqrt{s_{\mathrm{NN}}} \approx 7 \mathrm{GeV}$ and then does not change any more and is also compatible to the local slopes for $\mathrm{K}^{+}$from there on.

From the measured total yields of the strange particles the energy dependence of the number of produced strange quarks and anti-quarks can be constructed [11]. The strange quark carriers which were taken into account are $\mathrm{K}^{-}, \mathrm{K}^{0}, \Lambda$ (including $\left.\Sigma^{0}\right), \Xi^{0,-}, \Omega^{-}$, and $\Sigma^{ \pm}$. For the strange anti-quark these are $\mathrm{K}^{+}, \overline{\mathrm{K}}^{0}, \bar{\Lambda}$ (including $\bar{\Sigma}^{0}$ ), $\bar{\Xi}^{0,+}, \bar{\Omega}^{+}$, and $\bar{\Sigma}^{ \pm 1}$ The left panel of Fig. 5 shows the energy dependence of the normalized number of strange (anti-)quarks in comparison to the total number of pions $\left(\langle\pi\rangle=1.5\left(\left\langle\pi^{+}\right\rangle+\left\langle\pi^{+}\right\rangle\right)\right)$. While $\langle\pi\rangle /\left\langle N_{\mathrm{w}}\right\rangle$ is increasing with $\sqrt{s_{\mathrm{NN}}}$ rather smoothly, there is an indication for a change in the energy dependence of the total strangeness production around $\sqrt{s_{\mathrm{NN}}} \approx 7 \mathrm{GeV}$. The significance of this change can be visualized again by calculating the local slope, as defined in 2.1. While there is no change visible in the local slope for pions (see right panel of Fig. 5), it is significantly higher for (anti-)strangeness below $\sqrt{s_{\mathrm{NN}}} \approx 7 \mathrm{GeV}$ than above that energy, similar to the behaviour already present for $\mathrm{K}^{+}$.

\section{Transverse Mass Spectra}

The increase with energy of the inverse slope parameter $T$ of the kaon $m_{\mathrm{t}}$-spectra, as derived from an exponential fit, exhibits a sharp change to a plateau around $30 \mathrm{AGeV}$ [12, 13]. Since the kaon $m_{\mathrm{t}}$-spectra - in contrast to the ones of the lighter pions or the heavier protons - have to

\footnotetext{
${ }^{1}$ The $\mathrm{K}^{0}$ contribution was calculated using isospin symmetry $\left(\left\langle\mathrm{K}^{+}\right\rangle \approx\left\langle\mathrm{K}^{0}\right\rangle,\left\langle\mathrm{K}^{-}\right\rangle \approx\left\langle\overline{\mathrm{K}}^{0}\right\rangle\right)$. If no measurement is available, the values for the $\Xi$ and $\Omega$ yields were taken from statistical model fits $[8]$. The $\Sigma^{ \pm}$contribution was estimated based on the empirical factor $\left(\left\langle\Sigma^{ \pm}\right\rangle+\langle\Lambda\rangle\right) /\langle\Lambda\rangle=1.6$ [10]. Note that the strange quarks from the $\phi$ and $\eta$ are not included.
} 


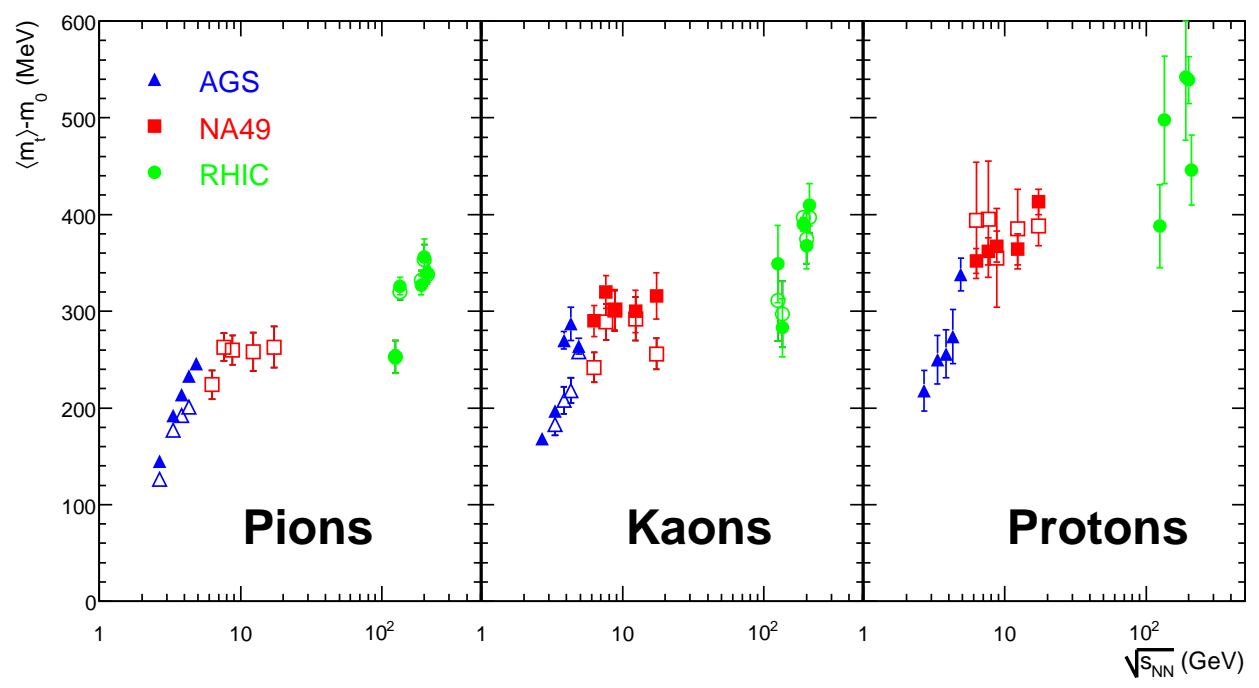

Figure 6: The energy dependence of $\left\langle m_{\mathrm{t}}\right\rangle-m_{0}$ for pions, kaons, and protons at mid-rapidity for 5 (10\%) most central $\mathrm{Pb}+\mathrm{Pb} / \mathrm{Au}+\mathrm{Au}$ reactions. Open symbols represent negatively charged particles [11].

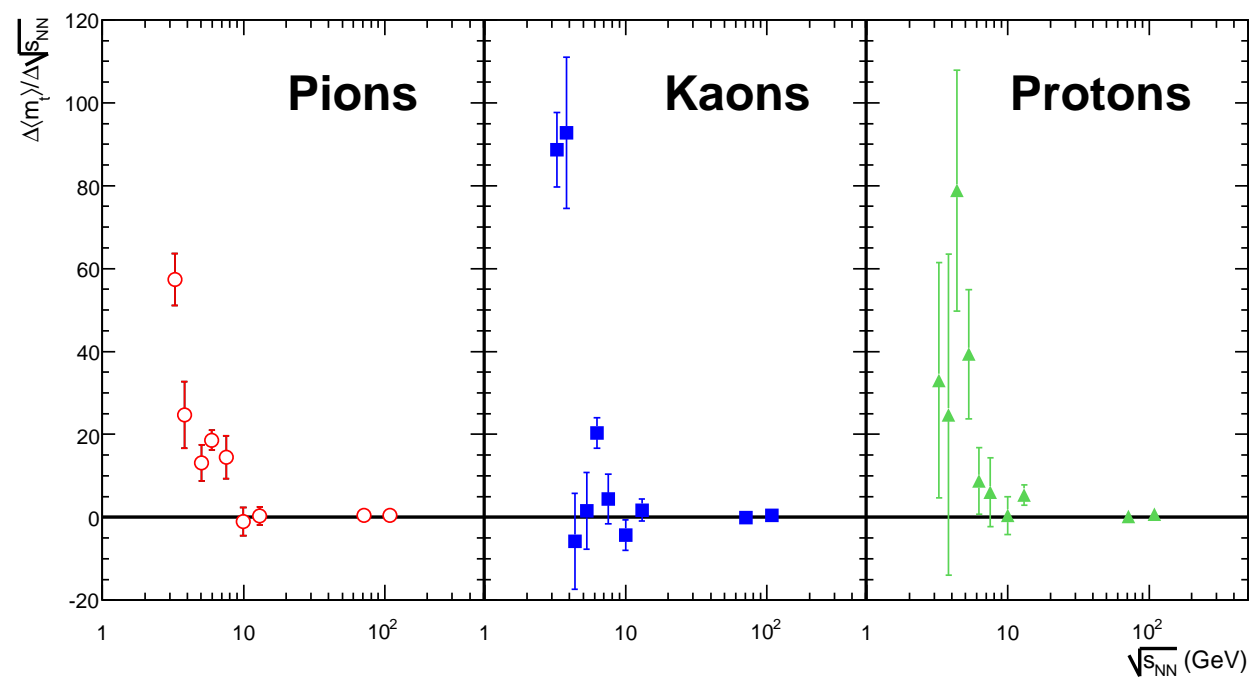

Figure 7: The local slope of the energy dependence of the mean transverse momentum $\Delta\left\langle m_{\mathrm{t}}\right\rangle / \Delta \sqrt{s_{\mathrm{NN}}}$ as a function of $\sqrt{s_{\mathrm{NN}}}$ for $\pi^{-}, \mathrm{K}^{+}$, and protons.

a good approximation an exponential shape, the inverse slope parameter provides in this case a good characterization of the spectra. For other particle species, however, the local slope of the spectra depends on $m_{\mathrm{t}}$. Instead, the first moment of the $m_{\mathrm{t}}$-spectra can be used to study their energy dependence. The dependence of $\left\langle m_{\mathrm{t}}\right\rangle-m_{0}$ on the center of mass energy $\sqrt{s_{\mathrm{NN}}}$ is summarized in Fig. 6. The change of the energy dependence around a beam energy of $20-30 \mathrm{AGeV}$ is clearly visible for pions and kaons. While $\left\langle m_{\mathrm{t}}\right\rangle-m_{0}$ rises steeply in the AGS energy range, the rise is much weaker from the low SPS energies on. To a lesser extent this change is also seen for protons. 

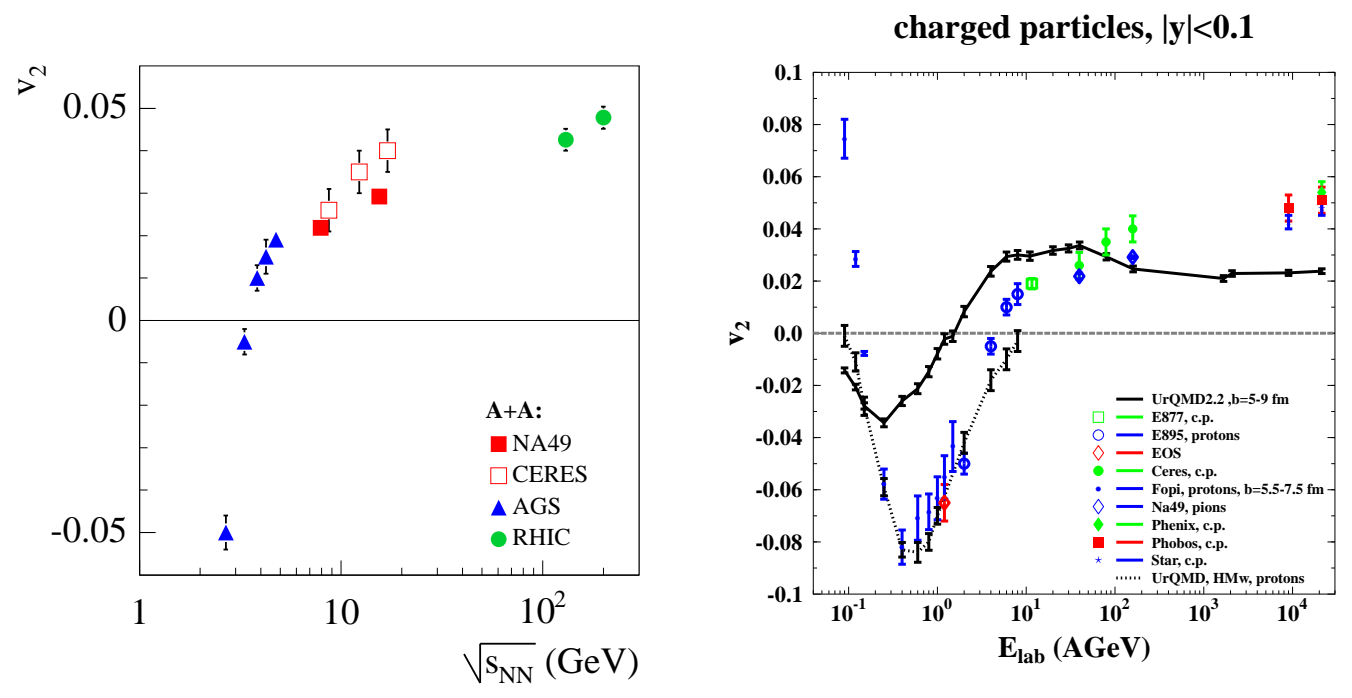

Figure 8: Left: The energy dependence of $p_{\mathrm{t}}$-integrated values of $v_{2}$ for pions at mid-rapidity [14, 15, 16, 17, 18, 19]. Right: The energy excitation function of elliptic flow of charged particles in $\mathrm{Au}+\mathrm{Au} / \mathrm{Pb}+\mathrm{Pb}$ collisions in mid-central collisions $(b=5-9 \mathrm{fm})$ with $|y|<0.1$ (full line) calculated with the UrQMD model [20]. This curve is compared to data from different experiments for mid-central collisions.

Again the structures in the energy dependence can be investigated more closely by calculation the local slope of the energy dependence of $\left\langle m_{\mathrm{t}}\right\rangle-m_{0}$. Figure 7 shows $\Delta\left\langle m_{\mathrm{t}}\right\rangle / \Delta \sqrt{s_{\mathrm{NN}}}$ as a function of $\sqrt{s_{\mathrm{NN}}}$. Generally, the local slope is higher at lower center-of-mass energies and decreases $\left(\sqrt{s_{\mathrm{NN}}} \leq 6-8 \mathrm{GeV}\right)$. It does not change any more when going to higher energies for all three particle species.

\section{Elliptic Flow}

An initial azimuthal anisotropy in space, as it is generated by the overlap region of two nuclei in non-central collisions, will be translated into a momentum anisotropy due to the different pressure gradients in the direction of the event plane and perpendicular to it. The measurement of the second harmonic of this momentum anisotropy, $v_{2}$, provides thus a measure of the initial pressure of the system. The energy dependence of $v_{2}$ is summarized in the left panel of Fig. 8. One observes a rapid rise from negative $v_{2}$ values at lower AGS energies to positive values, which changes its slope between top AGS and SPS energies. The further increase towards RHIC energies is then less pronounced. This measured energy dependence is compared to an UrQMD calculation in the right panel of Fig. 8, which is taken from [20]. While the energy dependence can roughly be described by this hadronic model at AGS energies and below, if a nuclear potential is included (dotted line), the model results in too small values for $v_{2}$ from SPS energies on (solid line). This might indicate that for the higher energies additional pressure from a partonic phase is needed in order to decribe the data. According to this comparison the partonic contribution would set in at SPS energies. An 
exact determination of the position of this onset would require more accurate data, since currently there is still a slight disagreement between the SPS data visible.

\section{Bose-Einstein Correlations}

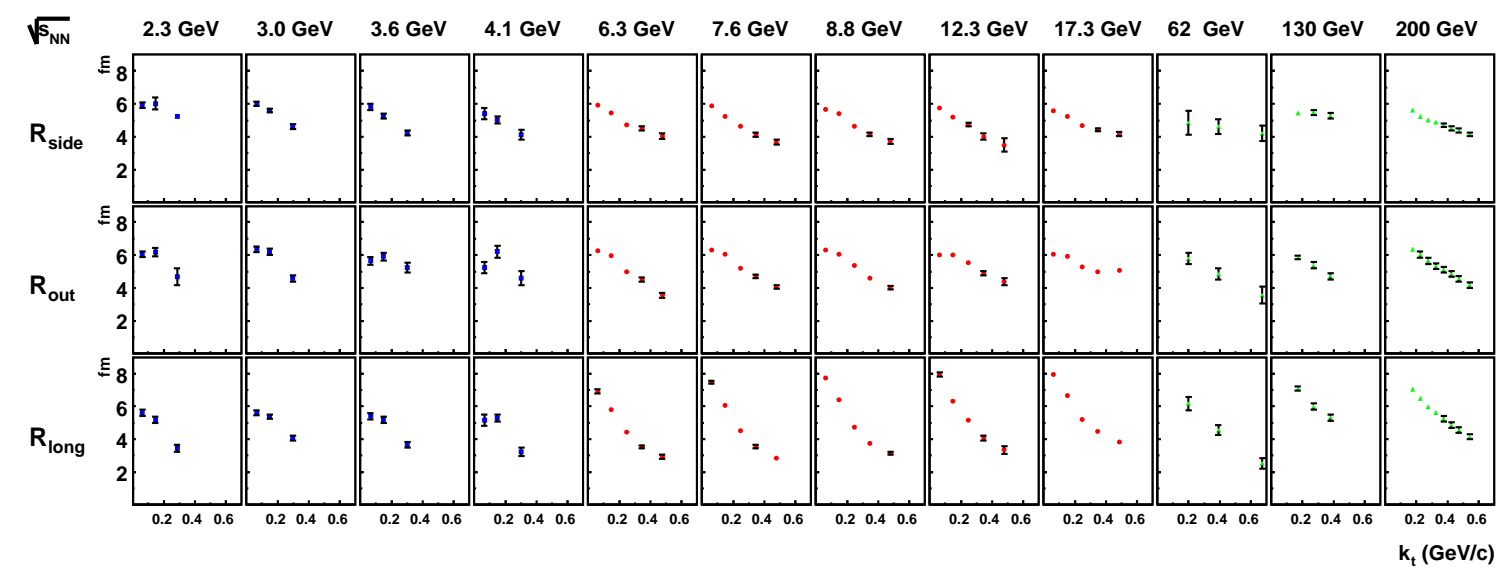

Figure 9: HBT radius parameters as a function of $k_{\mathrm{t}}$ measured by E895 [21], NA49 [25], PHOBOS [22], and STAR [23, 24].

Figure 9 shows a compilation of radius parameters measured in central nucleus nucleus collisions from AGS energies up to RHIC energies. One might expect that HBT radii should reflect also any change in the nature of the observed fireball, for instance due to a change of the lifetime of the system. However, even though other hadronic observable reveal some distinct features in their energy dependence, as discussed in the previous sections, structures in the energy dependence of HBT results are remarkably absent. This is exemplified in Fig. 10 where the $\sqrt{s_{\mathrm{NN}}}$-dependence of different radius parameters at a fixed $k_{\mathrm{t}}$ is shown. While there are indications for a slight variation at AGS energies, there is essentially no energy dependence any more at higher energies with the only exception of $R_{\text {long }}$, which slowly rises. However, there are still some unresolved discrepancies between NA49 [25] and NA45 [26], where the latter measurement would suggest an energy dependence of $R_{\text {out }}$ at the SPS.

\section{Summary}

The study of the energy dependence of various hadronic observables reveals several significant structures located in the energy region $\sqrt{s_{\mathrm{NN}}} \approx 6-8 \mathrm{GeV}$. There is an indication for a sudden change in the strangeness production and the $\left\langle m_{\mathrm{t}}\right\rangle-m_{0}$ of pions, kaons, and protons in this energy region, which can be identified by changes in the local slopes. Similar structures might also be present in the excitation function of elliptic flow, although here more measurements with higher 


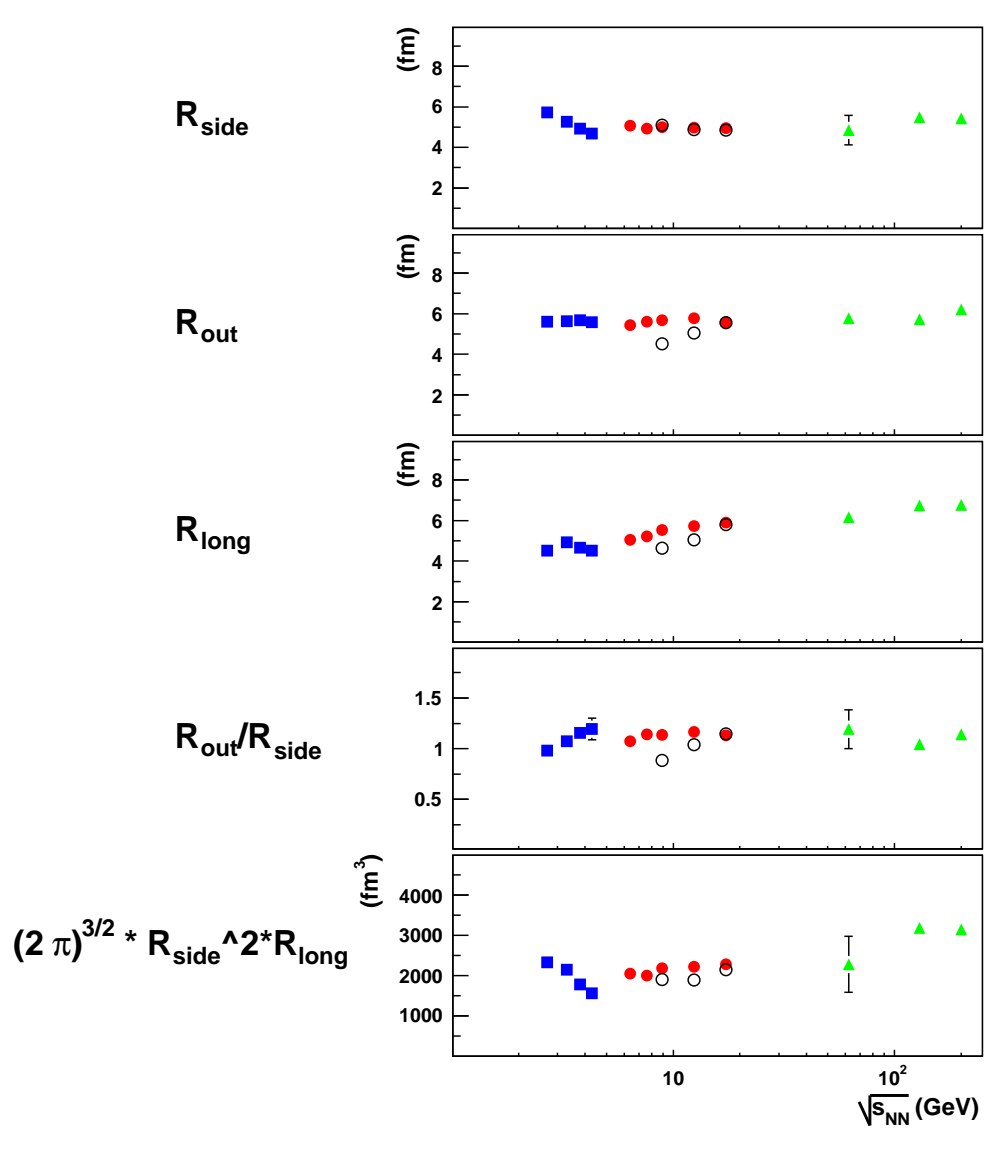

Figure 10: The dependence of the various radius parameters on the center-of-mass energy. The radii have been interpolated to $k_{\mathrm{t}}=0.2 \mathrm{GeV} / c$ [25].

precision would be needed. On the other hand, there is only a remarkably weak energy dependence of HBT-radii, with no clear signs of any structures. The change in the strangeness production translates itself into a sharp maximum in the $\left\langle\mathrm{K}^{+}\right\rangle /\left\langle\pi^{+}\right\rangle$ratio. Generally, the ratios of strange particles to pions are qualitativly described by a statistical hadron gas model, assuming full chemical equilibrium. However, a detailed comparison to the measurements shows that there are significant differences for $\sqrt{s_{\mathrm{NN}}}>7-8 \mathrm{GeV}$ which are about $3-5$ times larger than the total error. (kaons, $\phi$, and $\Xi$ ). However, in order to establish whether this indicates the onset of deconfinement, more measurement with high precision would be highly desirable. 


\section{References}

[1] M. Mitrovski et al. (for the NA49 collaboration), arXiv:nucl-ex/0606004.

[2] M. Gaździcki (for the NA49 collaboration), J. Phys. G 30, s701 (2004).

[3] M. Gaździcki and M.I. Gorenstein, Acta Phys. Polon. B 30, 2705 (1999).

[4] J. Cleymans, H. Oeschler, K. Redlich, and S. Wheaton, Eur. Phys. J. A 29, 119 (2006).

[5] M. Bleicher et al., J. Phys. G 25, 1859 (1999).

[6] E.L. Bratkovskaya et al., Phys. Rev. C 69, 054907 (2004).

[7] P. Braun-Munzinger, J. Cleymans, H. Oeschler, and K. Redlich, Nucl. Phys. A 697, 902 (2002).

[8] F. Becattini, M. Gaździcki, A. Keränen, J. Manninen, and R. Stock, Phys. Rev. C 69, 024905 (2004).

[9] P. Braun-Munzinger, J. Stachel, C. Wetterich, Phys. Lett. B 596, 61 (2004).

[10] A.K. Wróblewski, Acta Phys. Polon. B 16, 379 (1985).

[11] C. Blume (for the NA49 collaboration), J. Phys. G 31, s685 (2005).

[12] M.I. Gorenstein, M. Gaździcki, and K.A.Bugaev, Phys. Lett. B 567, 175 (2003).

[13] B. Mohanty, J. Alam, S. Sarkar, T.K. Nayak, and B.K. Nandi, Phys. Rev. C 68, 021901 (2003).

[14] C. Pinkenburg et al. (E895 collaboration), Phys. Rev. Lett. 83, 1295 (1999).

[15] J. Barrette et al. (E877 collaboration), Nucl. Phys. A 661, 329c (1999).

[16] J. Slivova et al. (NA45/CERES collaboration), Nucl. Phys. A 715, 615c (2003).

[17] C. Alt et al. (NA49 collaboration), Phys. Rev. C 68, 034903 (2003).

[18] C. Adler et al. (STAR collaboration), Phys. Rev. Lett. 87, 182301 (2001).

[19] J. Adams et al. (STAR collaboration), Phys. Rev. C 72, 014904 (2005).

[20] H. Petersen, Q. Li, X. Zhu, and M. Bleicher, arXiv:hep-ph/0608189.

[21] M. Lisa et al. (E895 collaboration), Phys. Rev. Lett. 84, 2798 (2000).

[22] B.B. Back et al. (PHOBOS collaboration), Phys. Rev. C 73, 031901(R) (2006).

[23] C. Adler et al. (STAR collaboration), Phys. Rev. Lett. 87, 082301 (2001).

[24] J. Adams et al. (STAR collaboration), Phys. Rev. C 71, 044906 (2005).

[25] S. Kniege et al. (NA49 collaboration), J. Phys. G 30, s1073 (2004).

[26] D. Adamova et al. (NA45/CERES collaboration), Nucl. Phys. A 714, 124 (2003). 